\title{
Efeitos do uso de gliricídia e rocha fosfatada no crescimento e nos teores de N, P e K nas culturas do quiabo e pepino
}

\author{
Effects of the use of gliricídia and phosphate rock on growth and N, P and $\mathrm{K}$ levels in okra and \\ cucumber crops \\ Efectos del uso de gliricídia y roca fosfórica sobre el crescimiento y los niveles de N, P y K em \\ cultivos de okra y pepino
}

Recebido: 10/05/2021 | Revisado: 16/05/2021 | Aceito: 22/05/2021 | Publicado: 08/06/2021

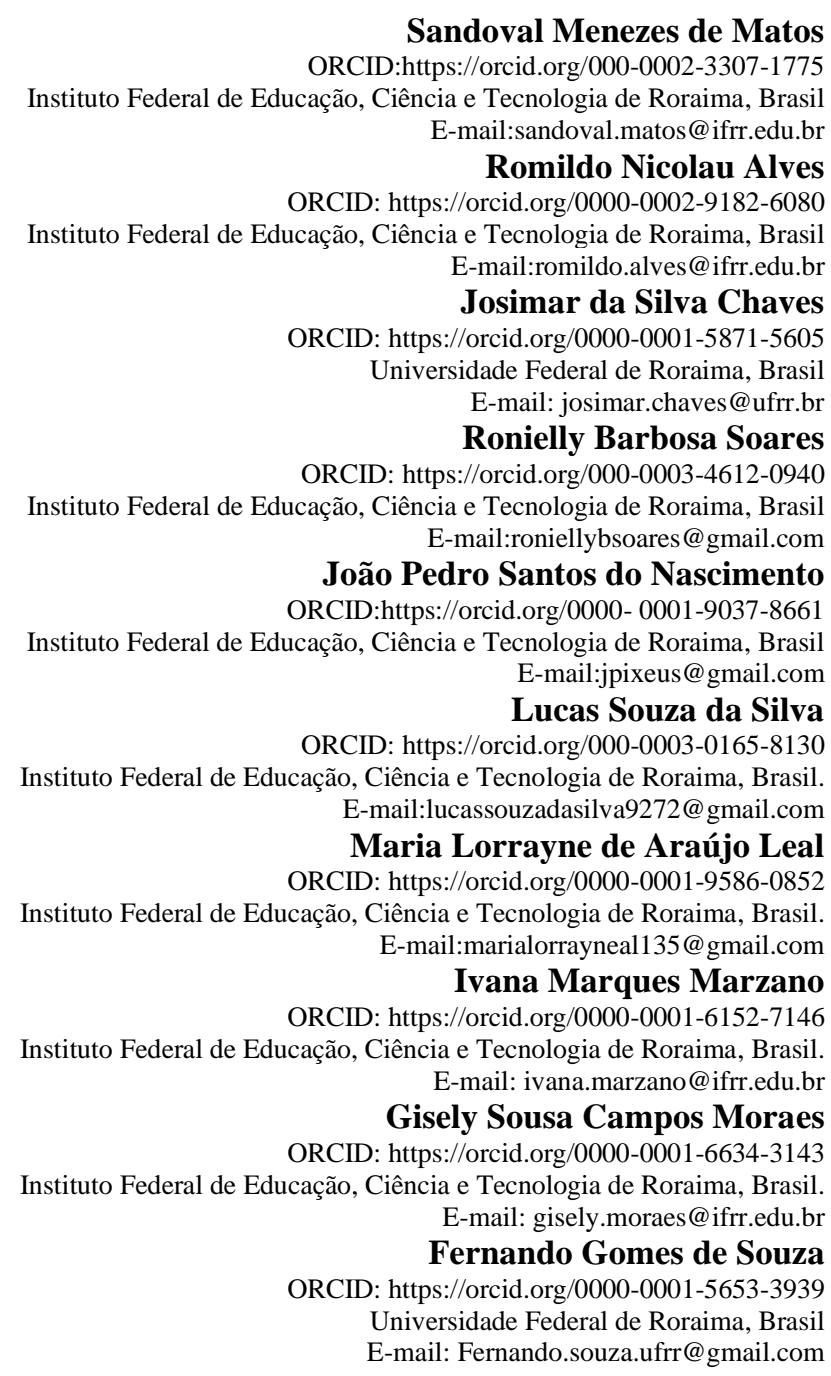

\section{Resumo}

As exigências nutricionais das hortaliças têm causado aos pequenos e médios produtores familiares prejuízos, com relação à redução da produtividade e a segurança alimentar da sociedade. Neste sentido, objetivou-se avaliar o desenvolvimento de plantas de quiabo (Abelmoschus escurentes L.) e de pepino (Cucumis sativus L.), utilizando esterco de ovinos associado com rocha fosfatada e a leguminosa gliricídia e a quantificação dos teores de $\mathrm{N}$, P e K nas estruturas das plantas. O experimento foi conduzido no Instituto Federal de Ciência e Tecnologia de Roraima Campus Novo Paraíso, em casa de vegetação, utilizando-se vasos com capacidade de $6 \mathrm{~L}^{-1}$, preenchidos com solo (Neosolo) coletado da camada de $0-20 \mathrm{~cm}$. O delineamento experimental foi em bloco ao acaso, com os tratamentos arranjados em fatorial 2x2, composto por dois níveis de gliricídia (sem gliricídia (S/G) e (com gliricídia (C/G) e, dois níveis de rocha fosfatada (sem rocha $(\mathrm{S} / \mathrm{R})$ e (com rocha $(\mathrm{C} / \mathrm{R})$, com quatro repetições, sendo distribuídos nos blocos 
de forma inteiramente casualizados. Foi adicionado aos vasos uma dose de esterco de ovinos, correspondente a $25 \mathrm{t}$ $\mathrm{ha}^{-1}$. As variáveis determinadas no estudo foram: altura de planta $(\mathrm{cm})$, espessura de caule $(\mathrm{mm})$, número de folhas, diâmetro do fruto $(\mathrm{mm})$, comprimento de fruto $(\mathrm{cm})$, peso de fruto $(\mathrm{g})$ e os teores de $\mathrm{N}$, P e K na planta e nos frutos. Os resultados encontrados para os diferentes tratamentos não apresentaram diferenças. Observou-se que a padronização da fertilidade dos vasos interferiu diretamente nos resultados estatísticos. Desta forma, os agricultores podem renunciar ao uso da folha da gliricídia e da rocha fosfatada, desde que ele tenha esterco de ovinos disponível.

Palavras-chave: Agricultura orgânica; Agroecologia; Sustentabilidade.

\begin{abstract}
The nutritional requirements of vegetables have caused losses to small and medium-sized family farmers in terms of reduced productivity and food security in society. In this sense, the objective was to evaluate the development of okra (Abelmoschus escurentes L.) and cucumber (Cucumis sativus L.) plants, using sheep manure associated with phosphate rock and gliricídia and the quantification of the levels of $\mathrm{N}, \mathrm{P}$ and $\mathrm{K}$ in plant structures. The experiment was conducted at the Federal Institute of Science and Technology of Roraima - Campus Novo Paraíso, in a greenhouse, using pots with a capacity of $6 \mathrm{~L}^{1}$, filled with soil (Neosolo) collected from the 0-20 $\mathrm{cm}$ layer. The experimental design was a randomized block, with treatments arranged in a $2 \times 2$ factorial, composed of two levels of gliricídia (without gliricídia (S / G) and (with gliricídia (C / G) and two levels of phosphate rock (without rock) (S / R) and (with rock $(\mathrm{C} / \mathrm{R})$, with four repetitions, being distributed in the blocks in a completely randomized way. A dose of sheep manure, corresponding to $25 \mathrm{t} \mathrm{ha}^{-1}$, was added to the pots. The variables determined in the study were: plant height $(\mathrm{cm})$, stem thickness $(\mathrm{mm})$, number of leaves, fruit diameter $(\mathrm{mm})$, fruit length $(\mathrm{cm})$, fruit weight $(\mathrm{g})$ and the contents of $\mathrm{N}, \mathrm{P}$ and $\mathrm{K}$ in the plant and in the fruits The results found for the different treatments showed no differences It was observed that the standardization of the fertility of the pots directly interfered in the statistical results, this way, the farmers can renounce the use of the leaf of the gliricídia and of the rock phosphate, as long as he has sheep manure available.
\end{abstract}

Keywords: Organic agriculture; Agroecology; Sustainability.

\title{
Resumen
}

Los requerimientos nutricionales de las hortalizas han causado pérdidas a los pequeños y medianos agricultores familiares en términos de reducción de la productividad y seguridad alimentaria de la sociedad. En este sentido, el objetivo fue evaluar el desarrollo de plantas de okra (Abelmoschus escurentes L.) y pepino (Cucumis sativus L.), utilizando estiércol de ovino asociado a roca fosfórica y gliricidia y la cuantificación de los niveles de N, P y K en planta. estructuras. El experimento se realizó en el Instituto Federal de Ciencia y Tecnología de Roraima - Campus Novo Paraíso, en invernadero, utilizando macetas con capacidad de 6 L-1 , llenas de tierra (Neosolo) recolectada de la capa de 0-20 cm. El diseño experimental fue un bloque al azar, con tratamientos dispuestos en un factorial $2 \times 2$, compuesto por dos niveles de gliricidia (sin gliricidia (S / G) y (con gliricidia (C / G) y dos niveles de roca fosfórica ( $\sin$ roca) $(\mathrm{S} / \mathrm{R})$ y (con piedra $(\mathrm{C} / \mathrm{R})$, con cuatro repeticiones, distribuyéndose en los bloques de forma completamente al azar. Se añadió a las macetas una dosis de estiércol de ovino, correspondiente a $25 \mathrm{t} \mathrm{ha}^{-1}$. Las variables determinadas en el estudio fueron: altura de la planta $(\mathrm{cm})$, grosor del tallo $(\mathrm{mm})$, número de hojas, diámetro del fruto $(\mathrm{mm})$, largo del fruto $(\mathrm{cm})$, peso del fruto $(\mathrm{g})$ y los contenidos de N, P y K en en la planta y en los frutos Los resultados encontrados para los diferentes tratamientos no mostraron diferencias Se observó que la estandarización de la fertilidad de las macetas interfirió directamente en los resultados estadísticos.

Palabras clave: Agricultura orgánica; Agroecología; Sustentabilidad.

\section{Introdução}

O Brasil tem se caraterizado como um dos grandes produtores mundiais de hortaliças. Segundo Cavalcante et al. (2010) e Paes et al. (2012), no Brasil, os maiores produtores de hortaliças são os pequenos e médios agricultores familiares. Visto que, esses produtores familiares, aproveitam todos os espaços das pequenas propriedades para trabalhar com a agricultura orgânica. Neste contexto, a agricultura orgânica possui papel de destaque no processo de produção dessas culturas.

Atualmente no cenário nacional, a produção de hortaliças em sistema orgânico, têm se apresentado como uma atividade em crescimento. De modo que, em decorrência da necessidade de se produzir hortaliças no sistema orgânico, se tornou uma realidade, levando qualidade de vida para a mesa dos consumidores e preservando os ambientes, dentro dos pequenos espaços cultivados. De acordo com Vidal (2011), o cultivo orgânico aparece não somente como uma forma alternativa ao sistema agroindustrial atual, mas, como uma forte base para uma mudança de paradigma e, da inter-relação entre a sociedade e a agricultura. Além do mais, vem resgatar as questões sociais, ecológicas e ambientais, colocando a agricultura 
dentro de um patamar sustentável, provocando um grande diferencial desse sistema de produção, pois, permite a equidade e o equilíbrio das relações sociais, bem como, a sua sustentabilidade no tempo e no espaço.

De acordo com Galati et al. (2013), o quiabo (Abelmoschus escurentes L.) e o pepino (Cucumis sativus L.), são hortaliças que fazem parte da cadeia produtiva de alimentos, devido à sua importância nutricional, uma vez que são importantes fontes de nutrientes para o homem. O quiabo é uma hortaliça muito popular em regiões de clima tropical, sobretudo devido à sua rusticidade e tolerância ao calor (Santos et al., 2020). O pepino, de acordo com Amaro et al. (2014), está entre as dez hortaliças de maior valor comercial no Brasil, sendo que, dentre as cucurbitáceas é uma das mais cultivadas em ambiente protegido. Essas culturas podem ser cultivadas tanto em modelo convencional como também no sistema orgânico.

O cultivo de hortaliças no Estado de Roraima, especialmente na região Sul do Estado, tem sido uma opção de renda para os pequenos e médios agricultores.

$\mathrm{Na}$ região Sul do Estado de Roraima, existem produtores que trabalham com hortaliças cultivadas em sistemas orgânicos como também os que aderem o modelo convencional. O uso de estratégias e técnicas, de cultivos e manejo em função de diferentes sistemas de manejo das culturas de forma eficiente e prático favorece o aumento da produtividade das hortaliças (Silva, 2016). Na região, os produtores já podem contar com o apoio de dois Núcleos de Estudos em Agroecologia, um núcleo no Instituto Federal de Educação Ciência e Tecnologia de Roraima - Campus Novo Paraíso, denominado NEPEAGRO (Núcleo de Estudo Pesquisa e, Extensão em Agroecologia) e, outro na Universidade Estadual de RoraimaUERR/Campus Rorainópolis. Os núcleos têm como objetivo desenvolver pesquisas que venham melhorar os sistemas produtivo dos agricultores familiares.

A utilização de fontes orgânicas e minerais de baixa solubilidade, como as rochas fosfatadas, são produtos preconizados para uso em sistemas agroecológicos. Neste sentido, a matéria orgânica proporciona melhorias nas propriedades químicas, físicas e biológicas dos solos. Dentre as propriedades físicas, destaca-se a melhora da densidade e a porosidade do solo favorecendo a retenção da umidade no solo. Em relação às propriedades químicas, pode-se citar o aumento da disponibilidade de nutrientes, (macro e micronutrientes) de forma lenta (Novais et al., 2007). Quanto as propriedades biológicas, o efeito está relacionado ao aumento da biodiversidade da população microbiana, favorecendo disponibilidade de nutrientes e contribuindo no processo de decomposição da matéria orgânica (Machado et al., 2012).

A Gliricídia sepium é uma leguminosa que tem sido plantada em propriedades rurais, pela sua alta capacidade de produzir biomassa, em condições de baixa disponibilidade hídrica com alto teor de nitrogênio $(\mathrm{N})$, em virtude de realizar o processo de fixação biológica de nitrogênio (FBN). Segundo Silva et al. (2020), as leguminosas fazem um papel especial no solo, como reduzindo o impacto ambiental, trabalhando a ciclagem de nutrientes evitando a erosão eólica e das chuvas, melhorando a macrofauna, aumentando os microrganismos do solo.

As rochas fosfatadas nos sistemas agroecológicos, entram como principal fonte de fósforo (P), visto que, além dos solos tropicais serem pobres em P, as fontes orgânicas em sua maioria contêm valores intermediários de P (Gomes, 2018, Pereira et al., 2020). Neste contexto, pesquisas sobre a disponibilidade de nutrientes a partir da junção de fontes orgânicas e fontes minerais de baixa solubilidade são de grande importância para os pequenos e médios agricultores do sistema orgânico.

Diante do exposto, objetivou-se neste trabalho avaliar o desenvolvimento vegetativo (altura de planta $(\mathrm{cm})$, espessura de caule $(\mathrm{mm})$, número de folhas, diâmetro do fruto $(\mathrm{mm})$, comprimento de fruto $(\mathrm{cm})$, peso de fruto $(\mathrm{g})$ ) e os teores de nutrientes (, P e K), na cultura do quiabo e pepino, utilizando esterco de ovinos associado com rocha fosfatada e a leguminosa gliricídia. 


\section{Metodologia}

Trata-se de uma pesquisa exploratória de natureza quantitativa (Pereira et al., 2018), realizado no Sul do estado de Roraima, conduzido no Instituto Federal de Educação Ciência e Tecnologia de Roraima - IFRR/Campus Novo Paraíso, em ambiente protegido, localizado nas coordenadas geográficas: latitude $1^{\circ} 15^{\prime} 01,46 \mathrm{~N}$ ', longitude $60^{\circ} 29^{\prime} 12,30 \mathrm{~W}$ ' e a uma altitude de 83,09 m, no município de Caracaraí - Roraima. O delineamento experimental foi em blocos casualizados, com os tratamentos distribuídos inteiramente ao acaso, com quatro repetições, em esquema fatorial 2 × 2, sendo dois níveis de gliricídia (sem gliricídia $-\mathrm{S} / \mathrm{G}\left(0 \mathrm{t} \mathrm{ha}^{-1}\right)$ e com gliricídia $\left.-\mathrm{C} / \mathrm{G}\left(20 \mathrm{tha}^{-1}\right)\right)$ e dois níveis de rocha fosfatada: sem rocha $-\mathrm{S} / \mathrm{R}(0$ $\left.\mathrm{kg} \mathrm{ha}^{-1} \mathrm{P}_{2} \mathrm{O}_{5}\right)$ e com rocha $-\mathrm{C} / \mathrm{R}\left(120 \mathrm{~kg} \mathrm{ha}^{-1} \mathrm{P}_{2} \mathrm{O}_{5}\right)$.

No estudo utilizou-se esterco de ovinos, gliricídia (Gliricídia sepium (Jacq.) Steud.) (folhas + pecíolo) e rocha fosfatada. O esterco de ovinos foi coletado em propriedade rurais no Município de Caracaraí - RR. As folhas e pecíolo de plantas de gliricídia foram coletadas no Núcleo de Estudos, Pesquisa e Extensão em Agroecologia NEPEAGRO/IFRR/Campus Novo Paraíso e a rocha fosfatada no mercado local, com as seguintes especificações: $28 \% \mathrm{P}_{2} \mathrm{O}_{5}$, segundo o fabricante. Uma sub amostra da rocha fosfatada, foi coletada e levada até o laboratório para determinação de sua composição química. Foi coletado, uma sub amostra do esterco e da gliricídia, e em seguida levadas ao Laboratório de Solo e Planta do IFRR/Campus Novo Paraíso; as mesmas foram pesadas para obter o peso úmido e logo após, colocadas em estufa de circulação forçada a $65^{\circ} \mathrm{C}$, por 72 horas. Após 72 horas foram trituradas e passadas em moinho tipo Willey, em seguida passado na peneira de $1 \mathrm{~mm}$ e tirado uma subamostra de $250 \mathrm{mg}$ para digestão.

Para a determinação de $\mathrm{N}$ total, amostras de $250 \mathrm{mg}$ foram digeridas utilizando $\mathrm{H}_{2} \mathrm{SO}_{4}+\mathrm{H}_{2} \mathrm{O}_{2}$ de acordo com Embrapa (2017). O C orgânico total foi determinado via úmida segundo Carmo e Silva (2012), o fósforo (P) por Colorimetria e o potássio (K) por fotometria de chama, de acordo com a Embrapa (2017).

Foram utilizados vasos de capacidade de $6 \mathrm{~L}^{-1}$ preenchidos com solo, classificados como Neosolo, coletados na camada (0-20cm), com as seguintes características químicas: $\mathrm{pH}$ em água $=5,4 ; \mathrm{Al}^{+3}=0,3 \mathrm{cmol}_{\mathrm{c}} / \mathrm{dm}^{3} ; \mathrm{Ca}^{+2}+\mathrm{Mg}^{+2}=0,3$ $\mathrm{cmol}_{\mathrm{c}} / \mathrm{dm}^{3} ; \mathrm{K}^{+}=0,04 \mathrm{cmol}_{\mathrm{c}} / \mathrm{dm}^{3}$ e P disponível $3,4 \mathrm{mg} / \mathrm{dm}^{3}$ de solo. Utilizou-se uma dosagem padrão de esterco de ovinos de $25 \mathrm{t} \mathrm{ha}^{-1}$ (Figueiredo et al. 2012) aplicada em todos os vasos. Os vasos foram umedecidos e deixados descansar por 10 dias. Após esse período, foi aplicada as doses de rocha fosfatada $\left(0\right.$ e $\left.120 \mathrm{~kg} \mathrm{ha}^{-1} \mathrm{P}_{2} \mathrm{O}_{5}\right)$ e a gliricídia $\left(0\right.$ e $\left.20 \mathrm{t} \mathrm{ha}^{-1}\right)$.

Foram semeadas três sementes por vaso de pepino (var. Valência) e quiabo (var. Aodai). Após a germinação, as plantas foram desbastadas, deixando uma planta/vaso. Os vasos foram irrigados com água destilada diariamente de acordo com a capacidade de campo, para evitar a salinidade do meio osmótico. As avaliações fitotécnicas iniciaram-se no $15^{\circ}$ dia após o semeio, sendo realizadas em intervalos de 2 dias até o final do experimento, aos 72 dias. No $35^{\circ}$ dia foram coletadas as folhas indicadoras (F.I) das culturas, colocadas em saco de papel, levadas ao laboratório e colocadas em estufa a $65{ }^{\circ} \mathrm{C}$ por $72 \mathrm{~h}$, de acordo com a recomendação da EMBRAPA (2017), com a finalidade de determinar a massa fresca e seca das folhas. Após realizar a quantificação foram trituradas no moinho e em seguida foi realizada a digestão para a análise de quantificação dos nutrientes no material. As variáveis avaliadas foram: altura de planta $(\mathrm{cm})$, espessura de caule $(\mathrm{mm})$, número de folhas, diâmetro do fruto $(\mathrm{mm})$, comprimento de fruto $(\mathrm{cm})$, peso de fruto $(\mathrm{g})$ e os teores de $\mathrm{N}, \mathrm{P}$ e K na planta e nos frutos.

As análises estatísticas foram feitas com auxílio do programa estatístico SISVAR versão 5.6 (Ferreira, 2014), aplicando-se o teste F para aferição de diferenças entre tratamentos. Foram realizados os desdobramentos utilizando-se teste de Tukey ao nível de $5 \%$ de probabilidade.

\section{Resultados e Discussão}

Os resultados das análises químicas dos teores de nutrientes das fontes orgânicas (folhas e pecíolos de gliricídia + esterco de ovinos) e mineral (rocha fosfatada) utilizadas, encontram-se na Tabela 1. A relação C/N é o parâmetro importante 
quando se estuda materiais orgânicos (Kiehl, 1995; Santos et al., 2008). Observa-se, que as fontes orgânicas (gliricídia (folhas + pecíolos) e esterco de ovinos), apresentaram relações C/N consideradas boas (Kiehl, 1995; Gomes, 2018; Pereira et al., 2020).

Tabela 1. Relação C/N, teores de macro e micronutrientes no esterco de ovino e gliricídia.

\begin{tabular}{|c|c|c|c|c|c|c|c|c|c|c|c|c|c|}
\hline FONTES & $\mathbf{C} / \mathbf{N}$ & $\mathbf{C}$ & $\mathbf{N}$ & $\mathbf{P}$ & $\mathbf{K}$ & $\mathbf{C a}$ & Mg & $\mathbf{S}$ & $\mathbf{C u}$ & $\mathbf{F e}$ & $\mathbf{Z n}$ & Mn & B \\
\hline & \multicolumn{8}{|c|}{ 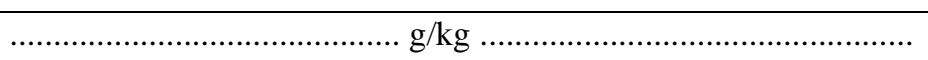 } & \multicolumn{5}{|c|}{ 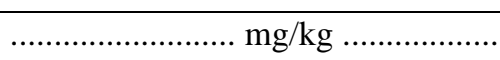 } \\
\hline Gliricídia & 12,79 & 430 & 33,60 & 2,10 & 20,50 & 11,00 & 5,55 & 2,50 & 4 & 144 & 14 & 34 & 57 \\
\hline Esterco ovino & 12,24 & 307 & 27,30 & 5,25 & 27,50 & 13,25 & 6,87 & 4,50 & 30 & 1380 & 160 & 385 & - \\
\hline Rocha fosfatada & - & - & - & 23,00 & 2,42 & 287,50 & 9,25 & 8,50 & 710 & 6100 & 10800 & 380 & - \\
\hline
\end{tabular}

Fonte: Laboratório Agrotécnico Piracicaba Ltda.

As fontes orgânicas são uma boa opção na produção de hortaliças, visto que apresentam bons teores de macro e micronutrientes (Kiehl, 1995). Os teores de N, P e K no esterco de ovinos (Tabela 1), foram superiores aos obtidos por Pereira et al. (2020), e igual ao obtido por Gomes (2018). Essas variações nos teores de N, P e K no esterco de ovinos estão relacionadas a qualidade nutricional dos alimentos fornecidos, a época de coleta do esterco e ao manejo do rebanho. A gliricídia apresentou valores próximos aos quantificados por Nascimento et al. (2021). Uma baixa relação C/N devido seu elevado teor de N. Esse elevado teor de N na gliricídia deve-se ao processo de fixação biológica de N (Souza et al., 2020). Pereira et al., (2020), ao analisarem gliricídia (Gliricídia sepium (Jacq.) Steud.) encontraram valores 2,8\% de N e 0,4\% de P, respectivamente, resultados semelhantes aos obtidos neste trabalho.

$\mathrm{O}$ desenvolvimento de uma planta requer uma sequência de eventos que deve ocorrer de forma precisa e ordenada. $\mathrm{O}$ crescimento é um termo quantitativo, relacionado a mudanças de tamanho e, ou massa (Taiz et al., 2017). Na Tabela 2, encontram-se as variáveis de altura de planta $(\mathrm{cm})$, diâmetro do caule $(\mathrm{mm})$, número de folhas, comprimento do fruto, peso do fruto e número de fruto/planta para a cultura do quiabo, nota-se que os tratamentos não se diferenciaram entre si pelo Tukey a 5\%. Estudos realizados por Neto e Tischer (2012), mostram que a falta dos macronutrientes nas hortaliças causam sintomas de deficiência na sua fisiologia vegetal, e, implicando no seu crescimento.

Quando avaliado os resultados da variável altura de planta $(\mathrm{cm}$ ), (Tabela 2), observou-se valor de 54,50 cm, este valor foi superior aos valores obtidos por Santos et al. (2010), que avaliaram o quiabeiro no início do florescimento, cultivado na presença de diferentes espécies de plantas competidoras e encontraram valores de altura de plantas variando de 12,42 a 21,87 cm; porém, inferior ao obtido por Costa (2014), que verificou a altura quiabeiro de 73,82 cm.

Em relação ao número de folhas, os resultados variaram de 9 a 11 folhas/planta, corroborando com os resultados obtidos por Santos et al. (2010), que avaliando o número de folhas de quiabeiro, quando cultivado com plantas competidoras, obtiveram valores variando de 8,67 a 10,92 folhas/planta.

Em relação à variável comprimento do fruto $(\mathrm{mm})$, os valores observados variaram de 140,3mm a 169,3 mm (Tabela 2), com média de 155,56 mm. Estudos de Oliveira et al. (2003), verificaram valores médio de 140,0 mm para o fruto de quiabo, quando se aplicou $200 \mathrm{~kg} \mathrm{ha}^{-1}$ de N; valor inferior ao obtidos neste estudo. Por outro lado, Folini e Zanin (1993), relatam valores de 111,0 a 150,0 mm de comprimento de fruto. Em relação ao peso do fruto $(\mathrm{g})$ no presente trabalho variou de 148,39g até 189,65g, com média de 166,21 g (Tabela 2), semelhantes ao obtidos por Mota et al. (2005), verificaram valores de $141,45 \mathrm{~g}$ a $173,17 \mathrm{~g}$, trabalhando com diferentes cultivares de quiabo. Em relação ao número de frutos/planta, os tratamentos apresentaram um valor médio de fruto de 10,50 frutos/planta (Tabela 2), o que corresponderia a uma produtividade de $17 \mathrm{t} \mathrm{ha}^{-1}$, 
que de acordo com Mota et al. (2000), a produtividade do quiabeiro, varia de 15.000 a $22.000 \mathrm{~kg} \mathrm{ha}^{-1}$. Esses resultados confirmam que a utilização de uma fonte orgânica, seja de origem vegetal e/ou animal na adubação da cultura é importante para manter a sua produtividade. Segundo Tokeshi et al. (2013), a presença dos macronutrientes e micronutrientes no solo, as plantas do quiabeiro terá um equilíbrio nutricional, elevando a sua produtividade.

Tabela 2. Variáveis fitotécnicas altura das plantas $(\mathrm{cm})$, diâmetro do caule $(\mathrm{mm})$, número de folhas, comprimento do fruto $(\mathrm{cm})$, peso dos frutos ( $\mathrm{g}$ ) e número de fruto/planta da cultura do quiabo.

\begin{tabular}{|c|c|c|c|c|c|c|c|c|c|c|c|c|}
\hline \multirow[t]{3}{*}{ Tratamentos } & \multicolumn{2}{|c|}{ Altura de planta } & \multicolumn{2}{|c|}{ Diâmetro do caule } & \multicolumn{2}{|c|}{$\begin{array}{c}\text { Número de } \\
\text { folhas }\end{array}$} & \multirow{2}{*}{\multicolumn{2}{|c|}{$\begin{array}{c}\begin{array}{c}\text { Comprimento de } \\
\text { fruto }\end{array} \\
\ldots \ldots . . . m m . . . . . . \\
\end{array}$}} & \multirow{2}{*}{\multicolumn{2}{|c|}{$\begin{array}{l}\text { Peso de fruto } \\
\ldots \ldots . . . \mathrm{g} . . . . . . . . \\
\end{array}$}} & \multirow{2}{*}{\multicolumn{2}{|c|}{$\begin{array}{c}\mathrm{N}^{\circ} \text { de fruto/planta } \\
-\end{array}$}} \\
\hline & \multicolumn{2}{|c|}{ 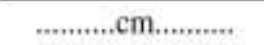 } & \multicolumn{2}{|c|}{$\ldots \ldots . . . . m m$} & \multicolumn{2}{|c|}{-} & & & & & & \\
\hline & S/R & $\mathrm{C} / \mathrm{R}$ & S/R & $\mathrm{C} / \mathrm{R}$ & S/R & $\mathrm{C} / \mathrm{R}$ & S/R & $C / R$ & $S / R$ & $\mathrm{C} / \mathrm{R}$ & $\mathrm{S} / \mathrm{R}$ & $\mathrm{C} / \mathrm{R}$ \\
\hline$S / G$ & $45,00 \mathrm{aA}$ & $46,88 \mathrm{aA}$ & $8,85 \mathrm{aA}$ & $10,68 a \mathrm{~A}$ & $9 \mathrm{aA}$ & $11 \mathrm{AA}$ & $169,3 \mathrm{Aa}$ & $160,8 \mathrm{Aa}$ & $189,65 \mathrm{Aal}$ & $169.7 \mathrm{Aa}$ & $11,50 \mathrm{Aa}$ & $16,08 \mathrm{Aa}$ \\
\hline $\mathrm{C} / \mathrm{G}$ & $51,75 \mathrm{aA}$ & $54,50 \mathrm{aA}$ & $11,66 \mathrm{aA}$ & $11,80 \mathrm{aA}$ & $10 \mathrm{aA}$ & $9 \mathrm{aA}$ & $140,3 \mathrm{Aa}$ & $152,1 \mathrm{Aa}$ & $148,39 \mathrm{Aai}$ & $157,1 \mathrm{Aa}$ & $8,75 \mathrm{Aa}$ & $15,21 \mathrm{Aa}$ \\
\hline Média geral & \multicolumn{2}{|c|}{49,53} & \multicolumn{2}{|c|}{10,75} & \multicolumn{2}{|c|}{12,68} & \multicolumn{2}{|c|}{155.56} & \multicolumn{2}{|c|}{166,21} & \multicolumn{2}{|c|}{10,50} \\
\hline $\mathrm{CV}(\%)$ & \multicolumn{2}{|c|}{7,80} & \multicolumn{2}{|c|}{5,07} & \multicolumn{2}{|c|}{4,89} & \multicolumn{2}{|c|}{7,66} & \multicolumn{2}{|c|}{16,11} & \multicolumn{2}{|c|}{9,84} \\
\hline
\end{tabular}

Médias com letras iguais na coluna e maiúsculas na linha não se diferenciam pelo teste de Tukey a 5\%. S/G: sem gliricídia; C/G: com gliricídia; S/R: sem rocha fosfatada; S/R: com rocha fosfatada Fonte: Elaborado pelos autores.

Em relação a cultura do pepino, verifica-se que as plantas atingiram em média $243 \mathrm{~cm}$ de altura, $9,19 \mathrm{~mm}$ de diâmetro de caule e 41,26 folhas (Tabela 3), valores superiores aos obtidos por Silva et al (2011). O tratamento C/G e C/R foi superior ao tratamento $\mathrm{C} / \mathrm{G}$ e S/R para variável altura de planta (Tabela 3). A superioridade desse tratamento deve-se basicamente ao efeito conjunto do esterco, gliricídia e da rocha fosfatada.

Tabela 3. Variáveis fitotécnicas altura das plantas (cm), diâmetro do caule (mm) e número de folhas da cultura do pepino.

\begin{tabular}{|c|c|c|c|c|c|c|c|c|c|c|}
\hline \multirow[t]{3}{*}{ Tratamentos } & \multicolumn{2}{|c|}{ Altura de planta } & \multicolumn{2}{|c|}{ Diâmetro do caule } & \multicolumn{2}{|c|}{$\begin{array}{l}\text { Número de } \\
\text { folhas }\end{array}$} & \multirow{2}{*}{$\begin{array}{c}\begin{array}{c}\text { Comprimento de } \\
\text { fruto }\end{array} \\
\ldots \ldots . \mathrm{cm} .\end{array}$} & \multicolumn{2}{|c|}{ Peso de fruto } & \multirow{2}{*}{$\begin{array}{c}\begin{array}{c}\mathrm{N}^{\circ} \mathrm{de} \\
\text { fruto/planta }\end{array} \\
-\end{array}$} \\
\hline & \multicolumn{2}{|c|}{ 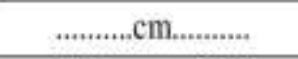 } & \multicolumn{2}{|c|}{ 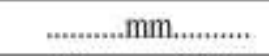 } & \multicolumn{2}{|c|}{-} & & \multicolumn{2}{|c|}{$\ldots \ldots \ldots$} & \\
\hline & $S / R$ & $\mathrm{C} / \mathrm{R}$ & $S / R$ & $C R$ & $S / R$ & $\mathrm{C} / \mathrm{R}$ & S/R $\quad C / R$ & $S / R$ & C/R & $\mathrm{C} / \mathrm{R}$ \\
\hline$S / G$ & $197,00 \mathrm{aA}$ & $232,75 \mathrm{aA}$ & $8,85 \mathrm{aA}$ & $7,79 \mathrm{aA}$ & $32 \mathrm{aA}$ & $46 \mathrm{aA}$ & $15,60 \mathrm{Aa} \quad 14,69 \mathrm{Aa}$ & 187,99Aa & $178,82 \mathrm{AaI}$ & $7,50 \mathrm{Aa} \quad 4,75 \mathrm{Aa}$ \\
\hline CG & $219,00 \mathrm{aB}$ & $317,25 \mathrm{aA}$ & $10,85 \mathrm{aA}$ & $9,70 \mathrm{aA}$ & $36 \mathrm{aB}$ & $48 \mathrm{aA}$ & $16,02 \mathrm{Aa} \quad 11,28 \mathrm{Aa}$ & $206,27 \mathrm{Aa}$ & $145,87 \mathrm{Ba}$ & $7,75 \mathrm{Aa} \quad 3,00 \mathrm{Aa}$ \\
\hline Média geral & \multicolumn{2}{|c|}{243,00} & \multicolumn{2}{|c|}{9,19} & \multicolumn{2}{|c|}{41,26} & 14,40 & \multicolumn{2}{|c|}{179,74} & 5,75 \\
\hline $\mathrm{CV}(\%)$ & \multicolumn{2}{|c|}{14,54} & \multicolumn{2}{|c|}{14,66} & \multicolumn{2}{|c|}{18,28} & 13,73 & \multicolumn{2}{|c|}{16,29} & 8,21 \\
\hline
\end{tabular}

Médias com letras iguais na coluna e maiúsculas na linha não se diferenciam pelo teste de Tukey a 5\%. S/G: sem gliricídia; C/G: com gliricídia; S/R: sem rocha fosfatada; S/R: com rocha fosfatada.

Fonte: Elaborado pelos autores.

O peso médio do fruto de pepino obtido no trabalho foi de 179,74g (Tabela 3), superior aos obtidos por Cardoso e Silva (2003), em duas épocas, no verão e no outono-inverno, variando entre 133,28 à 159,55g e 131,38 à $159,52 \mathrm{~g}$, respectivamente. Esses autores também quantificaram o número de fruto/planta, os quais foram superiores aos valores médios encontrados na presente pesquisa de 5,75. No cultivo de verão, eles verificaram valores de 18,40 a 25,42 fruto/planta e no outono-inverno os valores foram de 24,49 a 38,00 fruto/planta. O comprimento de frutos médio foi de 14,40 cm, valor inferior ao obtidos por Santi et al. (2013). Essas variações dos frutos são características influenciadas, além da adubação, pela genética e clima. 
Na Tabela (Tabela 4) estão apresentados os teores de N, P e K em diferentes partes do quiabeiro, onde observou se que os tratamentos não diferenciaram entre si pelo Tukey a 5\%, com exceção o N na folha. Para essa variável, os tratamentos C/G-S/R e C/G-C/R se diferenciaram estatisticamente pelo Tukey a 5\%. Vale destacar que, os teores de $\mathrm{N}$, P e $\mathrm{K}$ na folha indicadora foram determinados no momento do florescimento do quiabeiro e nas demais partes no final do experimento. Esse mesmo comportamento é observado para P e K. Considerando os teores de N, P e K na folha indicadora como um parâmetro de avaliação da qualidade nutricional do quiabeiro, nota-se que esses valores ficaram próximos ou dentro dos intervalos aceitáveis para o quiabo de acordo com os valores de referência indicados por Malavolta (1989); Pimentel (1985); Raij et al. (1997); Ribeiro et al. (1999), Zárate e Vieira (2018). O N na folha indicadora variou de 23,07 a 29,15 g kg-1 o P de 2,10 a 2,46

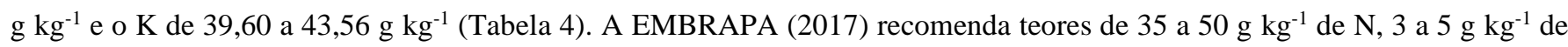
$\mathrm{P}$ e 25 a $40 \mathrm{~g} \mathrm{~kg}^{-1}$ de $\mathrm{K}$ para a cultura do quiabo.

Tabela 4. Teores dos nutrientes $\mathrm{N}, \mathrm{P}$ e K na cultura do quiabo.

\begin{tabular}{|c|c|c|c|c|c|c|c|c|c|c|c|c|}
\hline \multirow{3}{*}{ Tratamentos } & \multicolumn{2}{|c|}{$\mathrm{N}$ folha } & \multicolumn{2}{|c|}{$\mathrm{N}$ fruto } & \multicolumn{2}{|c|}{ P folha } & \multicolumn{2}{|c|}{ P fruto } & \multicolumn{2}{|c|}{$\mathrm{K}$ folha } & \multicolumn{2}{|c|}{$\mathrm{K}$ fruto } \\
\hline & \multicolumn{12}{|c|}{$\mathrm{g} \mathrm{kg}^{1}$} \\
\hline & S/R & $\mathrm{C} / \mathrm{R}$ & S/R & $\mathrm{C} / \mathrm{R}$ & S/R & $\mathrm{C} / \mathrm{R}$ & S/R & $\mathrm{C} / \mathrm{R}$ & S/R & $\mathrm{C} / \mathrm{R}$ & S/R & $\mathrm{C} / \mathrm{R}$ \\
\hline $\mathrm{S} / \mathrm{G}$ & $23,07 \mathrm{Aa}$ & $25,37 \mathrm{Aa}$ & $28,97 \mathrm{Aa}$ & $28,92 \mathrm{Aa}$ & $2,20 \mathrm{Aa}$ & $2,10 \mathrm{Aa}$ & $4,74 \mathrm{Aa}$ & $4,66 \mathrm{Aa}$ & $40,93 \mathrm{Aa}$ & $43,58 \mathrm{Aa}$ & $28,58 \mathrm{Aa}$ & $29,88 \mathrm{Aa}$ \\
\hline $\mathrm{C} / \mathrm{G}$ & $24,25 \mathrm{Ba}$ & $29,15 \mathrm{Aa}$ & $30.47 \mathrm{Aa}$ & $30,72 \mathrm{Aa}$ & $2,37 \mathrm{Aa}$ & $2,46 \mathrm{Aa}$ & $3,68 \mathrm{Aa}$ & 5.69Aa & $41,48 \mathrm{Aa}$ & $39,60 \mathrm{Aa}$ & $25,65 \mathrm{Aa}$ & $28,25 \mathrm{Aa}$ \\
\hline Média & \multicolumn{2}{|c|}{25,46} & \multicolumn{2}{|c|}{29,77} & \multicolumn{2}{|c|}{2,28} & \multicolumn{2}{|c|}{4,69} & \multicolumn{2}{|c|}{41,39} & \multicolumn{2}{|c|}{28,09} \\
\hline $\mathrm{CV}(\%)$ & \multicolumn{2}{|c|}{19,37} & \multicolumn{2}{|c|}{9,84} & \multicolumn{2}{|c|}{9,40} & \multicolumn{2}{|c|}{13,61} & \multicolumn{2}{|c|}{16,48} & \multicolumn{2}{|c|}{8,83} \\
\hline
\end{tabular}

Médias com letras iguais na coluna e maiúsculas na linha não se diferenciam pelo teste de Tukey a 5\%. S/G: sem gliricídia; C/G: com gliricídia; S/R: sem rocha fosfatada; S/R: com rocha fosfatada.

Fonte: Elaborado pelos autores.

Para a cultura do pepino, os resultados estatísticos para os teores de N, P e K nas folhas e nos frutos, nota-se que os tratamentos não se diferenciaram entre si (Tabela 5). Os valores médios dos teores na folha indicadora foram de $13,58 \mathrm{~g} \mathrm{~kg}^{-1}$ para o $\mathrm{N}, 1,84 \mathrm{~g} \mathrm{~kg}^{-1}$ para o P e 24,00 $\mathrm{g} \mathrm{kg}^{-1}$ para o $\mathrm{K}$. Observa-se que os teores de $\mathrm{N}$, P e $\mathrm{K}$ quantificados nas folhas foram inferiores aos valores recomendados por Solis (1982); Moltay et al. (1999); Ribeiro et al. (1999), Zárate e Vieira (2018). Solis (1982), trabalhando com a cultivar Aodai de pepineiro, cultivado a campo aberto, estabeleceu faixas adequadas de nutrientes para a cultura de 29 a 42; 2 a 4 e 16 a $27 \mathrm{~kg}^{-1}$ de massa seca foliar, para $\mathrm{N}, \mathrm{P}$ e $\mathrm{K}$, respectivamente. Moltay et al. (1999) propuseram como teores adequados de macronutrientes na cultura, 41; 5,2 e $45 \mathrm{~g} \mathrm{~kg}^{-1}$ de massa seca foliar para $\mathrm{N}$, P e K, respectivamente. Os valores de N e K, não diferiram aos recomendados por Maynard e Hochmuth (2007), que estabeleceram como faixas adequadas de macronutrientes para pepineiro cultivado em casa de vegetação as concentrações, em $\mathrm{g} \mathrm{kg}^{-1}$, de $25 \mathrm{a}$ 50; 5 a 10; 30 a 60; respectivamente para N, P e K. 
Tabela 5. Teores dos nutrientes N, P e K na cultura do pepino.

\begin{tabular}{|c|c|c|c|c|c|c|c|c|c|c|c|c|}
\hline \multirow{3}{*}{ Tratamentos } & \multicolumn{2}{|c|}{$\mathrm{N}$ folha } & \multicolumn{2}{|c|}{$\mathrm{N}$ fruto } & \multicolumn{2}{|c|}{ P folha } & \multicolumn{2}{|c|}{ P fruto } & \multicolumn{2}{|c|}{$\mathrm{K}$ folha } & \multicolumn{2}{|c|}{ K fruto } \\
\hline & \multicolumn{12}{|c|}{ (1) } \\
\hline & S/R & $\mathrm{C} / \mathrm{R}$ & S/R & $\mathrm{C} / \mathrm{R}$ & $\mathrm{S} / \mathrm{R}$ & C/R & $\mathrm{S} / \mathrm{R}$ & $\mathrm{C} / \mathrm{R}$ & S/R & $\mathrm{C} / \mathrm{R}$ & S/R & C/R \\
\hline$S / G$ & $13,65 \mathrm{Ba}$ & $20,50 \mathrm{Aal}$ & $22,30 \mathrm{Ai}$ & $18,30 \mathrm{Aa}$ & $1,76 \mathrm{Aa}$ & 1,81Ail & $6,98 \mathrm{Aa}$ & $5,63 \mathrm{Ail}$ & $22,40 \mathrm{Aa}$ & $33,90 \mathrm{Aa}$ & $45,35 \mathrm{Aa}$ & $38,23 \mathrm{Aal}$ \\
\hline CIG & $8,47 \mathrm{Aa}$ & $11,70 \mathrm{Ab}$ & $17,07 \mathrm{Aa}$ & $15,10 \mathrm{Aa}$ & $2,13 \mathrm{Aa}$ & $1,66 \mathrm{Aa}$ & $5,33 \mathrm{Aat}$ & $4,15 \mathrm{Aa}$ & $18,00 \mathrm{Aa}$ & $21,71 \mathrm{Aa}$ & $30,73 \mathrm{Aa}$ & $26,61 \mathrm{~A}$ \\
\hline Média & \multicolumn{2}{|c|}{13,58} & \multicolumn{2}{|c|}{$15,10 \mathrm{Aa}$} & \multicolumn{2}{|c|}{1,84} & \multicolumn{2}{|c|}{5,52} & \multicolumn{2}{|c|}{24,00} & \multicolumn{2}{|c|}{35,23} \\
\hline CV (\%) & \multicolumn{2}{|c|}{30,93} & \multicolumn{2}{|c|}{34,35} & \multicolumn{2}{|c|}{44,08} & \multicolumn{2}{|c|}{44,34} & \multicolumn{2}{|c|}{28,81} & \multicolumn{2}{|c|}{31,10} \\
\hline
\end{tabular}

Médias com letras iguais na coluna e maiúsculas na linha não se diferenciam pelo teste de Tukey a 5\%. S/G: sem gliricídia; C/G: com gliricídia; S/R: sem rocha fosfatada; S/R: com rocha fosfatada.

Fonte: Elaborado pelos autores.

A padronização da fertilidade do solo, foi possível verificar que a aplicação do esterco influenciou diretamente nos resultados obtidos e, observou-se que, a dose de $25 \mathrm{t} / \mathrm{ha}^{-1}$ de esterco de ovino atendeu a demanda nutricional das plantas em $\mathrm{N}$, $\mathrm{P}$ e K. No entanto, a dose de gliricídia e rocha fosfatada utilizada, pode ter ficado abaixo do que a planta precisava para uma elevada produtividade.

\section{Considerações Finais}

A aplicação do esterco de ovinos na dose de $25 \mathrm{t} \mathrm{ha}^{-1}$, influenciou diretamente nos resultados do trabalho suprindo a demanda nutricional da cultura do quiabo e do pepino.

Com a utilização de $25 \mathrm{t} \mathrm{ha}^{-1}$ de esterco de ovino, não há a necessidade, para a cultura do quiabo e pepino, da utilização de folha da gliricídia e da rocha fosfatada para atender às necessidades da planta.

Demais trabalhos que envolvam a temática de associação de fontes orgânicas e minerais na adubação de hortaliças para o a Amazônia devem ser desenvolvidos, para melhor utilização dos recursos disponíveis nas propriedades rurais desta região, haja vista a escassez de recursos financeiros pelos agricultores familiares. Perspectivas como outras fontes orgânicas para adubação e nível de adubação são atributos que podem ser analisados e vir a somar neste estudo.

\section{Agradecimentos}

Ao Instituto Federal de Educação, Ciência e Tecnologia de Roraima - IFRR/Campus Novo Paraíso e a Universidade Estadual de Roraima -UERR; ao Programa de Pós-graduação em Agroecologia pelo apoio no desenvolvimento da Dissertação de Mestrado. E ao Núcleo de Estudo, Pesquisa, Extensão em Agroecologia (NEPEAGRO) pela disponibilização do espaço para montagem do experimento.

\section{Referências}

Amaro, A. C. E., Macedo, A. C., Ramos, A. R. P., Goto, R., Ono, E. O., \& Rodrigues, J. D. (2014). The use of grafting to improve the net photosynthesis of cucumber. Theoretical and Experimental Plant Physiology, v.26, (3), 241-249, 10.1007/s40626-014-0023-1.

Cardoso, A. I. I., \& Silva, N. S. (2003). Avaliação de híbridos de pepino tipo japonês sob ambiente protegido em duas épocas de cultivo. Revista Horticultura Brasileira. 21: 171-176.

Carmo, D. L., \& Silva, C. A. (2012). Métodos de quantificação de carbono e matéria orgânica em resíduos orgânicos. Revista Bras. Ciência do Solo. 36:12111220 .

Cavalcante, L. F., Diniz, A. A., Santos, L. C. F., Rebequi, A. M., Nunes, J. C., \& Brehm, M. A. D. S. (2010). Teores foliares de macronutrientes em quiabeiro cultivado sob diferentes fontes e níveis de matéria orgânica. Ciências Agrárias, Londrina, v. 31, (1), 19-28.

Costa, R. A. (2014). Cultura do quiabo submetida a lâminas de irrigação por gotejamento em função da evaporação em tanque classe A44f. Tese (Doutorado em Agronomia/Irrigação e Drenagem) - Faculdade de Ciências Agronômicas da Unesp, Campus de Botucatu, Botucatu - SP. 
Dias, V. P., \& Fernandes, E. (2006). Fertilizantes: uma visão global sintética: BNDES Setorial. 138 p.

Empresa Brasileira de Pesquisa Agropecuária - EMBRAPA. (2017). Manual de métodos de análise de solo. (3ª ed.) Brasília/DF, 575p.

Ferreira, D. F. (2014). Sisvar: um guia dos seus procedimentos de comparações múltiplas Bootstrap. Ciência e Agrotecnologia, 38, (2), 109-112.

Filgueira, F. A. R. (2012). Novo manual de olericultura: Agrotecnologia moderna na produção e comercialização de hortaliças. (3ª ed.) Viçosa: UFV. 421 p.

Folini, E. F., \& Zanin, C. W. (1993). Classificação dos frutos do quiabeiro em ensaios de competição de cultivares. Horticultura Brasileira. Brasília. 11: 162170 .

Galati, V. C., Filho, A. B. C., \& Alves, A. U. (2013). Crescimento e acúmulo de nutrientes da cultura do quiabeiro. Semina: Ciências Agrárias, Londrina, 34, (1), 191-199.

Gomes, G. C. (2018). Produção e qualidade de compostos orgânicos no sul do Estado de Roraima. Dissertação (Mestrado em Agroecologia), Universidade do Estado de Roraima - UERR, Boa Vista (RR), 59 p.

Kiehl, J. E. (1985). Fertilizantes orgânicos. Piracicaba: Agronômica Ceres, 492 p.

Machado, D. M., Schossler, T. R., Zuffo, A. M., Andrade, F. R., \& Piauilino, A. C. (2012). Atividades microbianas e as transformações no ciclo dos elementos no solo. Enciclopédia Biosfera, Centro Científico Conhecer, Goiânia, v.8, (15), 27 - 32.

Malavolta, E. (1989). Avaliação do estado nutricional das plantas: princípios e aplicações. Piracicaba: Associação Brasileira para Pesquisa da Potassa e do Fosfato, 201p.

Maynard, D. N., \& Hochmuth, G. J. (2007). Knott's Handbook for Vegetable Growers. John Wiley \& Sons, Inc: New Jersey.

Moltay, I., Soyergin, S., Surmeli, N., Genç, Ç., \& Yurekturk, M. (1999). Determination of the nutrient status of greenhouse-grown cucumbers (Cucumis sativus L.) in the east Marmara region. Acta Horticulturae, v.492, (10), 237-243.

Mota, W. F., Finger, F. L., \& Casali, V. W. D. (2000). Olericultura: Melhoramento Genético do Quiabeiro. Viçosa: UFV, 144 p.

Mota, W. F., Finger, F. L., Silva, D. J. H., Corrêa, P. C., Firme, L. P., \& Neves, L. L. M. (2005). Caracterização físico-química de frutos de quatro cultivares de quiabo. Revista Horticultura Brasileira. v.23: 722-725.

Nascimento, J. P. S., Alves, R. N., Chaves, J. S., Matos, S. M., Soares, R. B., Silva, L. S., Leal, M. L. A., Silva, H. S., \& Lima, F. S. (2021). Teores de macronutrientes e produção de biogás a partir da co-digestão de fontes orgânicas. In: Silva Sousa, C., Sousa, F. \& Cotrim, S. (Org.). Agroecologia: Métodos e Técnicas para uma Agricultura Sustentável. (1ªed.): Editora Científica, 3, 30-42, 10.37885/210303486.

Neto, M. S., \& Tischer, J. (2012). Avaliação da deficiência de macronutrientes em alface crespa. Ensaios e Ciência: Ciências Biológicas, Agrárias de Saúde. $16,(2), 43-57$.

Novais, R. F., Smyth, T. J., \& Nunes, F. N. Fósforo. In: Novais, R. F. et al (Ed.). (2007). Fertilidade do solo. Viçosa: Sociedade Brasileira de Ciência do Solo, p. $471-550$

Oliveira, A. P., Alves, A. U., Dornelas, C. S. M., Silva, J. A., Pôrto, M. L., \& Alves, A.U. (2003). Rendimento de quiabo em função de doses de nitrogênio. Acta Scientiarum Agronomy, 25, (2), 265-268.

Paes, H. M. F., Esteves, B. D. S., \& Souza, E. F. D. (2012). Determinação da demanda hídrica do quiabeiro em Campos dos Goytacazes, RJ. Revista Ciência Agronômica, Fortaleza, 43, (2), 256-261.

Pereira A. S. et al. (2018). Metodologia da pesquisa científica. [free e-book]. Santa Maria/RS. Ed. UAB/NTE/UFSM.

Pereira, H. R., Alves, R. N., Chaves, J. S., Nascimento, J. P. S., Soares, R. B., \& Souza, L. S. (2020). Compostagem de serragem e adubação da cultura da abóbora no Sul do estado de Roraima. Research, Society and Development, 9, 1-18, 10.33448/rsd-v9i11.9510.

Pimentel. A. A. M. P. (1985). Olericultura no trópico úmido. (1 $1^{\mathrm{a}}$ ed.): Editora Agronômica Ceres, 322p.

Raij, B. V., Cantarella, H., Quaggio, J. A., \& Furlani, A. M. C. (1997. Recomendações de Adubação e Calagem para o Estado de São Paulo, (2ª ed.) rev. ampl. Campinas, Instituto Agronômico \& Fundação IAC, 285 p. (Boletim Técnico, 100).

Ribeiro, A. C., Guimarães, P. T. G., \& Alvarez, V. V. H. (eds.) (1999). Recomendações para o uso de corretivos e fertilizantes em Minas Gerais $5^{a}$ aproximação. Viçosa - Comissão de Fertilidade do Solo do Estado de Minas Gerais, 359 p.

Santi, A., Scaramuzza, W. L. M. P., Soares, D. M. J., Scaramuzza, J. F., Dallacort, R., Krause, W., \& Tieppo, R. C. (2013). Desempenho e orientação do crescimento do pepino japonês em ambiente protegido. Horticultura Brasileira, 31: 649-653.

Santos, E. A., Vale, L. S. R., Oliveira, H. F. E. de., Miranda, T. M., Mello, C. E. L., Souza, A. D. V. de., \& Leal, V. N. (2020). Quality of okra seeds produced under different irrigation depths. Research, Society and Development, [S. 1.], 9, (11), 37 - 42, 10.33448/rsd-v9i11.10184.

Santos, J. B., Silveira, T. P., Coelho, P. S., Costa, O. G., Matta, P. M., Silva, M. B., \& Drumond Neto, A. P. (2010). Interferência de plantas daninhas na cultura do quiabo. Revista Planta Daninha. 28: 255-262.

Silva, A. S., Silva, I. F., Silva Neto, L. F., \& Souza, C. (2011). Semeadura direta na produção do milho em agricultura de sequeiro na região nordeste do Brasil. Ciência Rural, Santa Maria, 41, (9), p. 1556-1562. 
Research, Society and Development, v. 10, n. 6, e50810615939, 2021

(CC BY 4.0) | ISSN 2525-3409 | DOI: http://dx.doi.org/10.33448/rsd-v10i6.15939

Silva, D. A. (2016). Estratégia de Agricultura de Precisão para Definição de Planos de Manejo Inteligente. Dissertação (Mestrado em Agronomia), Universidade de Santa Maria - Frederico Westphalen.

Solis, F. A. M. (1982). Concentração e extração de nutrientes e distúrbios nutricionais na cultura de pepino (Cucumis sativus L.) var. Aodai. (1982). 139f. Tese (Doutorado em Agronomia) - Escola Superior de Agricultura "Luiz de Queiroz", Universidade de São Paulo, Piracicaba.

Taiz, L., Zeiger, E., Moller, I. M., \& Murphy, A. (2017). Fisiologia e Desenvolvimento Vegetal. Artmed.

Vidal, M. C. (2011). Cultivo Orgânico de Hortaliças. In: Congresso Brasileiro de Olericultura, 51. Horticultura Brasileira 29. Viçosa: MG.

Zárate, N. A. H., \& Vieira, M. C. (Org.). (2018). Hortas: conhecimentos básicos. (1ª ed.) - Dourados, MS: Seriema, 298 p. 